\title{
OBSERVATION OF NONTHERMAL EMISSION FROM THE SUPERNOVA REMNANT IC 443 WITH RXTE
}

\author{
S. J. Sturner ${ }^{1,2}$, J. W. Keohane ${ }^{3}$, and O. Reimer ${ }^{4}$ \\ 1 Universities Space Research Association, 7501 Forbes Blvd., Suite 206, Seabrook, \\ MD 20706-2253, USA \\ ${ }^{2}$ Laboratory for High Energy Astrophysics, Code 661, NASA Goddard Space Flight Center, \\ Greenbelt, MD 20771, USA \\ 3 North Carolina School of Science and Mathematics, Durham, NC 27715-2418, USA \\ 4 Ruhr-University, Bochum, Germany
}

\begin{abstract}
In this paper we present analysis of X-ray spectra from the supernova remnant IC 443 obtained using the PCA on $R X T E$. The spectra in the $3-20 \mathrm{keV}$ band are well fit by a two-component model consisting of thermal and nonthermal components. We compare these results with recent results of other X-ray missions and discuss the need for a cut-off in the nonthermal spectrum. Recent Chandra and XMM-Newton observations suggest that much of the nonthermal emission from IC 443 can be attributed to a pulsar wind nebula. We present the results of our search for periodic emission in the RXTE PCA data. We then discuss the origin of the nonthermal component and its possible association with the unidentified EGRET source.
\end{abstract}

\section{INTRODUCTION}

The supernova remnant (SNR) IC $\left.443\left(1=189.1^{\circ}\right\}, b=+3.0^{\circ}\right)$ is a canonical mixed-morphology SNR. It is classified as a shell-type remnant in the radio, but is center filled with thermal X-ray emitting gas. Braun and Strom (1986) characterized the complex morphology of IC 443 as a series of three interconnected shells, but subsequently, Asaoka and Aschenbach (1994), using ROSAT data, determined that one of these subshells was in fact an older foreground SNR, G189.6+3.3. The complex morphological structure of IC 443 can to a large extent be attributed to interactions with local molecular clouds. The SNR shock - molecular cloud interactions of IC 443 have been studied in detail by mapping line emission from shocked species such as $\mathrm{H}_{2}$, $\mathrm{CO}$, and $\mathrm{HCO}+$ (see e.g. Cesarsky et al. 1999; Rho et al. 2001; Tauber et al. 1994).

IC 443 has been found to belong to a select subclass of SNRs that exhibit non-thermal X-ray emission (Bocchino and Bykov 2000, 2001; Olbert et al. 2001; Keohane et al. 1997; Wang et al. 1992) which includes, e.g., Cas A (Allen et al. 1997), SN 1006 (Koyama et al. 1995), and G347.3-0.5 (Slane et al. 1999). Wang et al. (1992) found that the remnant-integrated $2-20 \mathrm{keV}$ spectrum observed with the Ginga LAC could be well fit by a two-component model. They found that a thermal plus power-law model with a temperature of 0.68 $\mathrm{keV}$ and a spectral index of 2.2 (with a $90 \%$ confidence range of $2.07-2.33$ ) provided a good fit to the data but they also found that a two-temperature model provided an acdeptable fit. Using spatially-resolved spectra taken with the $A S C A$ GIS, Keohane et al. (1997) found that $\sim 60 \%$ of the remnant-integrated $7 \mathrm{keV}$ emission could be attributed to 2 unresolved sources in the south central region and eastern rim of the remnant. Ratio maps showed that these regions had significantly harder spectra than the rest of the SNR. Spectral analysis of the brighter of the two sources found that its spectrum was well described by a two-component model containing a thermal component with a temperature of $0.89 \mathrm{keV}$ and a power-law component with index $2.3 \pm 0.2$. These sources were further examined using the MECS and PDS on BeppoSAX by Bocchino and Bykov (2000) who found that the spectrum of the brighter source was well fit by a power law with index 1.96 $(+0.21,-0.12)$. Olbert et al. (2001), using Chandra and VLA data, have argued that the brighter of the two sources was a pulsar wind nebular (PWN), CXOU J061705.3+222127. Using the high sensitivity of XMMNewton, Bocchino and Bykov (2001) showed that the emission of the synchrotron nebula softened from a 
power-law index of 1.63 at its core to 2.30 at its edge. This spectral softening is in accord with expectations. for a PWN in which the emitting electrons lose energy as they propagate away from the pulsar.

IC 443 is also interesting because there is a spatially-coincident unidentified high-energy $(>100 \mathrm{MeV})$ gamma-ray source $\left(3 E G \mathrm{~J} 0617+2238 ; 1=189.00^{\circ}, \mathrm{b}=+3.05^{\circ}\right)$ which was discovered by the EGRET experiment on the Compton Gamma Ray Observatory (CGRO) (Hartman et al. 1999; Sturner and Dermer 1995; Sturner et al. 1996; Esposito et al. 1996). Most models for the gamma-ray source have concentrated on locally accelerated cosmic rays interacting with the nearby molecular clouds (e.g. Sturner et al. 1997; Baring et al. 1999). The discovery of possible PWN within IC 443 has thrown some doubt on that explanation.

\section{OBSERVATIONS}

We observed SNR IC 443 with the PCA on the RXTE between August 13 and 16, 1996 with the $\sim 1^{\circ}$ field of view (FOV) centered at $\alpha=6^{\mathrm{h}} 17^{\mathrm{m}} 1.5^{\mathrm{s}} \delta=+22^{\circ} 34^{\prime} 52.3^{\prime \prime}(\mathrm{J} 2000)$. The observation consisted of seven segments with a net exposure time of 35,488 seconds. Data was collected in both Standard-2 and GoodXenon modes. The Standard-2 mode data consists of pulse-height histograms with 129 channel spectra with 16 second time resolution. Data from this mode were used exclusively for spectral analysis. GoodXenon mode data consists of binary events words that have a time stamp resolution of $\sim 1 \mu$ s and utilize the entire 256 channel pass band of the PCA. We used the GoodXenon data for timing analysis.

Analysis of the data was performed using the standard suite of FTOOLS. We used the background model developed for faint sources, L7-240, which has recently been revised to a "CM" version. Use of this new background model significantly impacts the fitting results. A more detailed description of the analysis method and the background model can be found at http://heasarc.gsfc.nasa.gov/docs/xte/xhp_proc_analysis.html.

\section{SPECTRAL ANALYSIS}

As we described in the introduction, IC 443 has a very complex morphology, particularly in low-energy X-rays (e.g. Asaoka and Aschenbach 1994; Keohane et al. 1997). The spectrum we derive here is that of the spatially integrated X-ray emission from the entire SNR (the PCA $\sim 1^{\circ}$ FWHM field-of-view is larger than the angular extent of the SNR, $\sim 45^{\prime}$ ) and is therefore the superposition of spectra from regions with widely disparate conditions. Thus analysis and interpretation of the RXTE PCA spectrum is complicated. Previous authors have performed detailed analysis of spatially-resolved, low-energy X-ray $(\leq 10 \mathrm{keV})$ spectra with higher spectral resolution than possible with the PCA. We can make no improvement on these results here. We instead concentrate on the area where $R X T E$ can make a significant contribution, characterization of the nonthermal component and the search for evidence of a spectral cutoff at energies $>10 \mathrm{keV}$. Interpretation of the nonthermal spectrum is also complicated by source superpositon but to a much lesser extent than the nonthermal emission since it has been shown that the nonthermal emission, at least at energies $<10 \mathrm{keV}$, is concentrated in two compact regions of IC 443 (Bocchino and Bykov 2000; Keohane et al. 1997).

Analysis of the nonthermal spectrum requires an accurate characterization of the thermal component. We fit the combined data from all PCUs and all observation segments in XSPEC using two-component models that included both thermal and nonthermal components. We found that the generalized nonequilibrium ionization model (GNEI) of Borkowski et al. (2001) best characterized the low-energy thermal component of the spectrum. This model, in a simplified manner, takes into account the temperature evolution of the SNR when determining ionization fractions and transition rates. We explored fitting the high-energy component with power-law, broken power-law, and cutoff power-law models. As stated above, the version of the background model used during the fitting process has a large effect on the fitting results. We will compare and contrast the results obtained using both the older L7-240 model and its newer CM version. This CM revision of the model is purported to have less unmodelled variance, i.e. smaller systematic errors, than previous models (Markwardt 2002).

First, we will discuss the results using the older background model. We find no indication for a break or cut off in the nonthermal spectrum using this background model. The best-fit $\chi^{2}$ values are almost identical with 234.06 for 357 degrees of freedom (dof), 233.67 for 355 dof, and 234.13 for 356 dof for the power-law, broken power-law, and cut off power-law models, respectively. Comparing the power-law and broken powerlaw results, an FTEST indicates that the slight improvement in fit obtained by adding a break to the power-law does not warrant the decrease in the number of degrees of freedom. Hence there is no indication of a break or cut-off in the nonthermal spectrum using this background model. The best fit parameters for the GNEI + power-law model are $\mathrm{kT}=0.60(+0.02,-0.04) \mathrm{keV}$ and photon power-law index $\alpha=2.22 \pm 0.16$, where the errors are $90 \%$ confidence. This spectral index is consistent with the remnant-integrated spectral index found 
by Wang et al. (1992), using the Ginga LAC, of $2.20 \pm 0.13$. It is also consistent with the indices derived by both Keohane et al. (1997) and Bocchino and Bykov (2000) for the proposed synchrotron nebula within IC $443,2.3 \pm 0.2$ and $1.96(+0.21,-0.12)$ respectively. But it must be emphasized that the high quality data in these two cases is limited to energies $\leq 10 \mathrm{keV}$. The ionization timescale, $\tau$, and the ionization-timescale averaged plasma temperature, $\langle\mathrm{kT}\rangle$, are not well constrained by the data, but have best-fit values of $\tau=17.4$ $(+24.24,-4.17) \mathrm{kyr} \mathrm{cm}^{-3}$ and $<\mathrm{kT}>=1.95(+0.98,-0.18) \mathrm{keV}$.

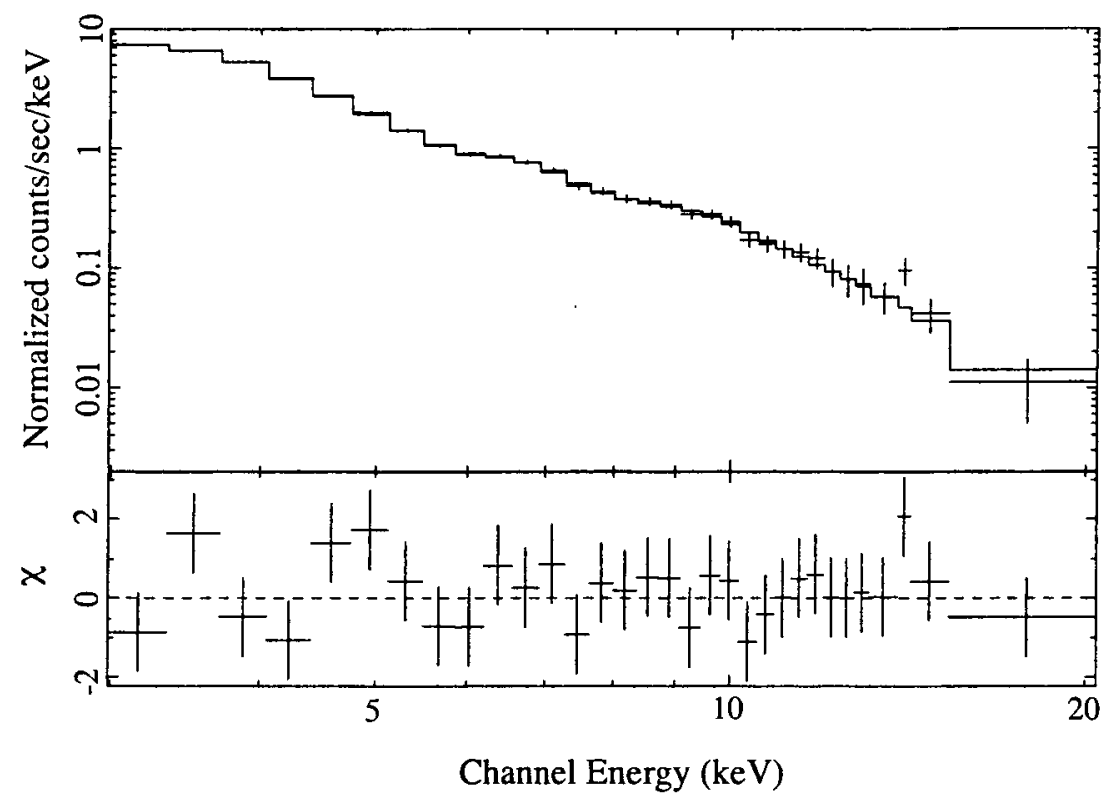

Fig. 1. The background subtracted data using the CM background model and the folded best fit GNEI + broken power-law model (see text for parameter values). Also shown are the residuals in units of $\sigma$.
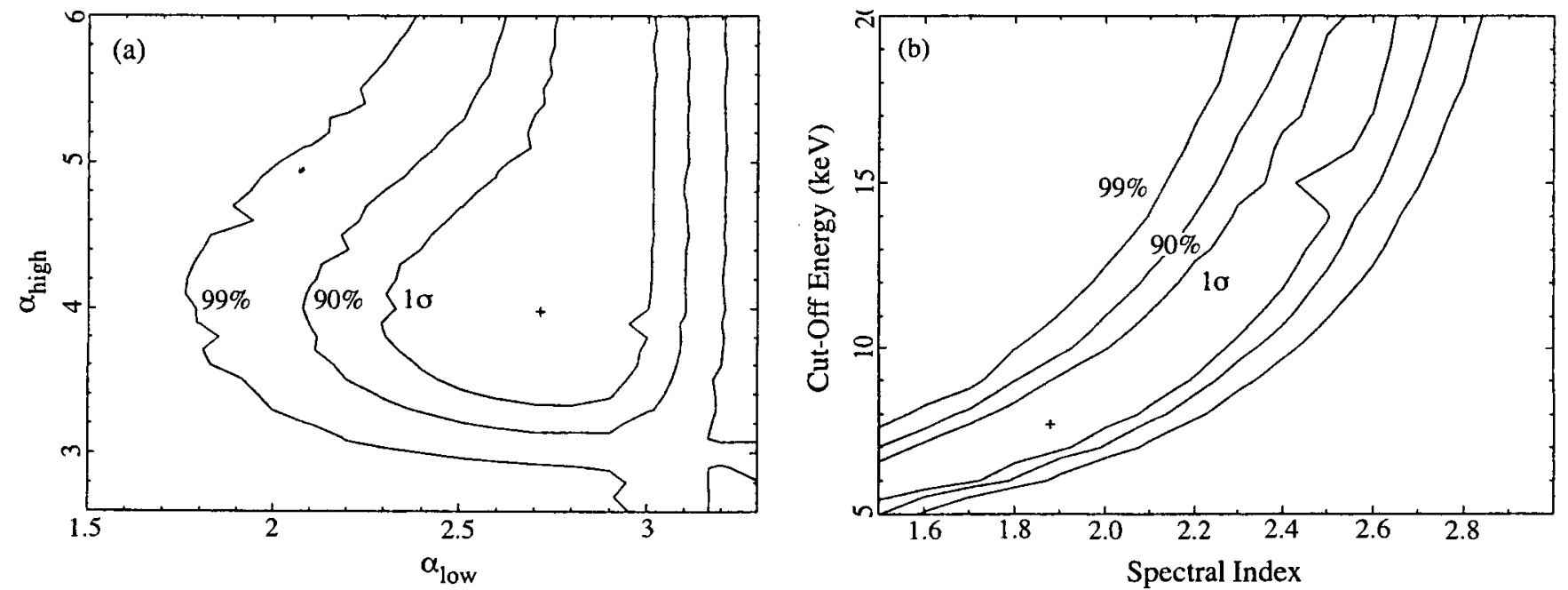

Fig. 2. In (a) we show the allowed values for the broken power-law spectral indices at $10,90 \%$, and $99 \%$ confidence levels. In (b) we show the allowed values for the spectral index and cut-off energy for the cut-off power-law model. Best fit parameters are indicated by the + .

The results are significantly different when the CM version of the background model is used. The best-fit $\chi^{2}$ values are 214.50 for 322 dof and 202.30 for 320 dof for the power-law and broken power-law models, respectively, see Figure 1. Again using an FTEST to ascertain the need for a break to the power-law, we find a probability of $8.53 \times 10^{-5}$, indicating that the addition of a break in the power-law is justified. For the case of a broken power-law, the best-fit GNEI model parameters show only small variations from the best fit using the older background model. The best fit parameters are: $\mathrm{kT}=0.56(+0.10,-0.03) \mathrm{keV}, \tau=12.51(+17.21,-7.12)$ $\mathrm{kyr} \mathrm{cm}^{-3}$, and $<\mathrm{kT}>=2.00(+1.26,-0.27) \mathrm{keV}$. The nonthermal model best-fit parameters are: $\alpha_{\text {low }}=2.72$, $\alpha_{\text {high }}=3.99$, and $E_{\text {break }}=9.90$ but they are not well constrained. The $1 \sigma, 90 \%$, and $99 \%$ confidence regions 
for the spectral indices are shown in Figure 2a. Note that the indices found by the previous authors mentioned above fall just within the $90 \%$ confidence contour.

The cut off power-law model was problematic to fit to the data using the new background model. The data could be adequately fit, $\chi^{2} \sim 205$ for 321 dof, using a wide range of model parameter values and with only small changes to $\chi^{2}$, i.e. $\Delta \chi^{2}<1$. Specifically, flattening of the spectral index could be compensated for by a decrease in the cut-off energy. This is illustrated in Figure $2 \mathrm{~b}$ where we show that spectral indices from $<1.5$ to $\sim 2.6$ are within the 10 confidence region for that model parameter if the cut-off energy is allowed to be as low as $5 \mathrm{keV}$. This requirement for a break or cutoff below $20 \mathrm{keV}$ appears to disagree with the Ginga results (Wang et al. 1992) although the fact that a two-temperature model fit provided an acceptable fit to the data suggests that curvature of the high-energy Ginga spectrum cannot be ruled out. The $A S C A, B e p p o S A X$, and $X M M-N e w t o n$ results indicate that the break or cut-off energy cannot be below $\sim 10 \mathrm{keV}$. If the cut-off energy is restricted to be $>10 \mathrm{keV}$, then, as seen in Figure $2 \mathrm{~b}$, the index must then be greater than $\sim 2$.

The $2-10 \mathrm{keV}$ integrated flux for the GNEI + broken power-law model is $6.3 \times 10^{-11} \mathrm{erg} \mathrm{cm}^{-2} \mathrm{~s}^{-1}$. Keohane et al. (1997) found that the integrated $2-10 \mathrm{keV}$ flux within their two GIS pointings, which they estimate to $\geq$ $90 \%$ of the total remnant flux, to be $5 \pm 1 \times 10^{-11} \mathrm{erg} \mathrm{cm}^{-2} \mathrm{~s}^{-1}$, consistent with our current findings.

\section{TIMING ANALYSIS}

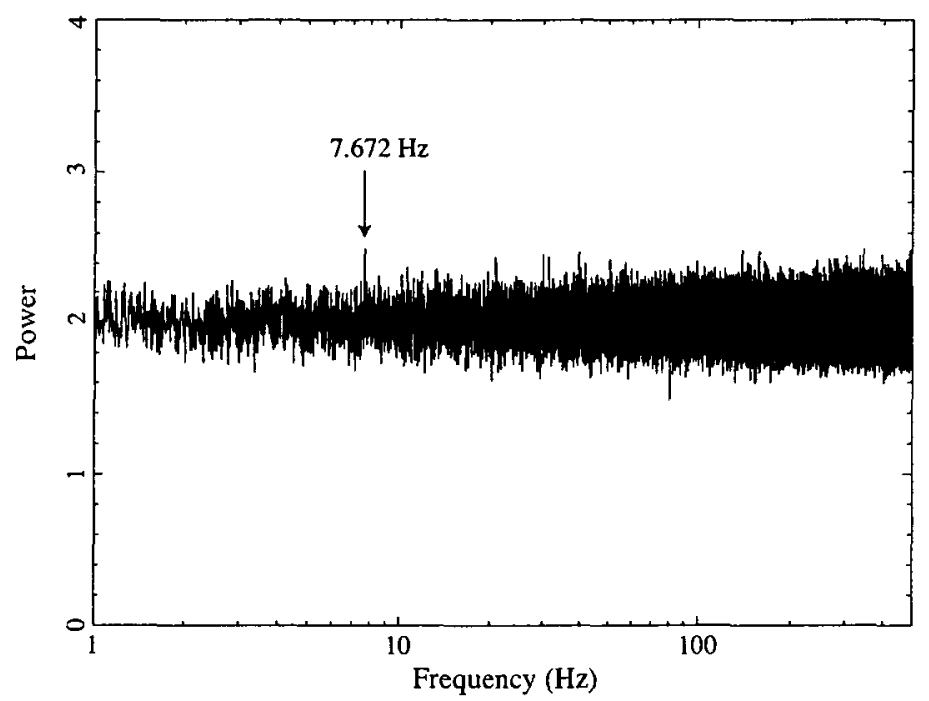

Fig. 3. Here is the derived power spectrum for IC 443. The fequemcy of maximum power I $7.672 \mathrm{~Hz}$ which would correspond to a pulsar period of $130 \mathrm{msec}$.

The recent work of Olbert et al. (2001) has indicated that much of the non-thermal emission seen by $A S C A$ and BeppoSAX originates from the proposed PWN CXOU J061705.3+222127. The excellent timing resolution and sensitivity of the PCA provides an opportunity to search for the pulsar powering the nebula. The event data were barycenter corrected and FFTs were performed on the entire dataset using the XRONOS application POWSPEC. We found no evidence for a pulsar with a frequency in the range $1-500 \mathrm{~Hz}$. The derived power distribution reproduced the theoretically expected distribution to a high degree of accuracy. Given our chosen normalization and the number of intervals that the data were divided into (329), the power distribution should closely approximate a Gaussian centered at a power of 2.0 with $\sigma=0.1103$. The measured standard deviation of the distribution was 0.1118 , see Figure 3. The maximum power was 2.4944 at $7.672 \mathrm{~Hz}$. The measured power required to surpass a detection confidence threshold of $(1-\varepsilon)$ is given by

$$
\frac{\varepsilon}{N_{\text {bins }}} \approx \frac{1}{\sqrt{2 \pi}} \int_{\frac{p_{\text {det }}-2}{\sigma}}^{\infty} \exp \left(\frac{-t^{2}}{2}\right) d t
$$

where $\mathrm{N}_{\text {bins }}$ is the number of bins in the power spectrum and $\mathrm{P}_{\text {det }}$ is the power needed to surpass the given confidence threshold. The $90 \%$ and $99 \%$ confidence threshold powers are 2.5146 and 2.5645 , respectively, in excess of our maximum measured power. 
The EGRET experiment on the CGRO has found a $17.4 \sigma$ source that is spatially consistent with IC 443, 3EG J0617+2238 (Hartman et al. 1999; Sturner and Dermer 1995; Sturner et al. 1996; Esposito et al. 1996). The source shows very marginal variability (Tomkins 1999) which suggests that it is unlikely to be a background blazar. This lack of variability and its positional coincidence with IC 443 have led to postulations that this source is due either to locally accelerated cosmic rays (Sturner et al. 1997; Gaisser et al. 1998; Baring et al. 1999; Bykov et al. 2000) or a pulsar (Yadigaroglu and Romani 1995).

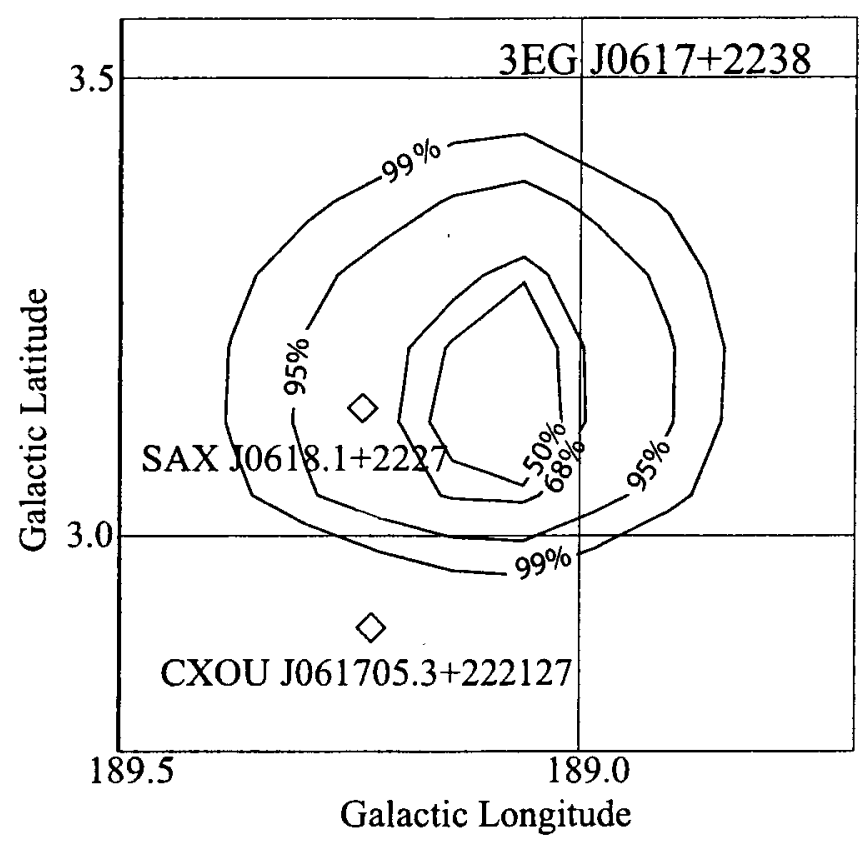

Fig. 4. The confidence contours for the location of the unidentified EGRET source 3EG J0617+2238 as well as the locations of the two hard X-ray sources SAX J0618.1+2227 and CXOU J061705.3+222127.

We plot the EGRET position contours for 3EG J0617+2238 in Figure 4 for photon energies $>100 \mathrm{MeV}$. Also shown are the locations of two hard X-ray sources observed by ASCA (Keohane et al. 1997), BeppoSAX (Bocchino and Bykov 2000) and XMM-Newton (Bocchino and Bykov 2001). These two sources are thought to produce the majority of the hard X-ray emission observed from IC 443 (Keohane et al 1997). Can any firm conclusions be drawn about an association between either of these X-ray sources and the EGRET source? The answer is no. There is better positional agreement between the EGRET source and the BeppoSAX source than with the Chandra source but this is a weak measure of association because of the difficulties in generating a position for the EGRET source given its proximity to the Crab and Geminga as well as the local bright Galactic plane emission. There also needs to be drawn a distinction between a positional agreement and a counterpart identification. Counterpart identification requires positional agreement with an identified source, a plausible gamma-ray emission mechanism, and consistent multifrequency behavior (both temporal and spectral). CXOU J061705.3+222127 seems a more likely counterpart based on the known classes of unambiguously identified EGRET sources which include pulsars. This conclusion is also dangerous considering the lack of concrete knowledge about SAX J0618.1+2227. Closer examination of the multiwavelength emission from SAX J0618.1+2227 is required before eliminating it as a possible counterpart to the EGRET source.

\section{CONCLUSIONS}

We find that when using the somewhat older source background model for the RXTE PCA, the nonthermal portion of the data was well fit a powerlaw that extended beyond $20 \mathrm{keV}$ with a spectral index in agreement with previous authors. In contrast, when using the newer CM version of the background model, the nonthermal portion of the data required a cut-off or broken powerlaw with a cut-off or break energy near 10 $\mathrm{keV}$. Whether or not this result is in disagreement with the Ginga results (Wang et al. 1992) is uncertain as a two-temperature thermal model also provided an acceptable fit to their data. We conducted a search for evidence of coherent pulsations in the data and found none at greater than $90 \%$ confidence. 


\section{REFERENCES}

Allen, G. E., et al., Evidence for X-ray synchrotron emission from electrons accelerated to $40 \mathrm{TeV}$ in the supernova remnant Cassiopeia A,, $A p J, 487$, L97-L100, 1997.

Asaoka, L., and B. Aschenbach, An X-ray study of IC 443 and the discovery of a new supernova remnant by ROSAT, $A \& A, 284,573-582,1994$.

Baring, M. G., D. C. Ellison, S. P. Reynolds, I. A. Grenier, and P. Goret, Radio to gamma-ray emission from shell-type supernova remnants: predictions from nonlinear shock acceleration models, $\mathrm{ApJ}, \mathbf{5 1 3}, 311$ $338,1999$.

Bocchino, F., and A. M. Bykov, The plerion nebula in IC 443: the XMM-Newton view, $A \& A, 376,248-253$, 2001.

Bocchino, F., and A. M. Bykov, Hard X-ray emission from IC 443: evidence for a shocked molecular clump?, $A \& A, 362$, L29-L32, 2000.

Borkowski, K. J., W. J. Lyerly, and S. P. Reynolds, Supernova remnants in the sedov expansion phase: thermal $\mathrm{X}$-ray emission, $A p J, 458,820-835,2001$.

Braun, R., and R. G. Strom, The structure and dynamics of evolved supernova remnants. The IC 443 complex, $A \& A, 164,193-207,1986$.

Bykov, A.M., R. A. Chevalier, D. C. Ellison, and Y. A. Uvarov, Nonthermal emission from a supernova remnant in a molecular cloud, $A p J, 538,203-216,2000$.

Cesarsky, D., P. Cox, G. Pineau des Forets, E. F. van Dishoeck, F. Boulanger, F., and C. M. Wright, ISOCAM spectro-imaging of the $\mathrm{H}_{2}$ rotational line in the supernova remnant IC 443, $A \& A, 348,945-949,1999$.

Esposito, J. A., S. D. Hunter, G. Kanbach, and P. Sreekumar, EGRET observations of radio-bright supernova remnants, ApJ, 461, 820-827, 1996.

Gaisser, T. K., R. J. Protheroe, and T. Stanev, Gamma-ray production in supernova remnants, ApJ, 492, 219 $227,1998$.

Hartman, R. C., et al., The third EGRET catalog of high-energy $\gamma$-ray sources, ApJS, 123, 79-202, 1999.

Keohane, J. W., R. Petre, E. V. Gotthelf, M. Ozaki, and K. Koyama, A possible site for cosmic ray acceleration in the supernova remnant IC 443, ApJ, 484, 350-359, 1997.

Koyama, K., R. Petre, E. V. Gotthelf, M. Matsuura, M. Ozaki, and S. S. Holt, Evidence for shock acceleration of high-energy electrons in the supernova remnant SN 1006, Nature, 378, 255-258, 1995.

Markwardt, C., private communication, 2002.

Olbert, C. M., C. R. Clearfield, N. E. Williams, J. W. Keohane, and D. A. Frail, A bow shock nebula around a compact X-ray source in the supernova remnant IC 443, ApJ, 554, L205-L208, 2001.

Rho, J., T. H. Jarrett, R. M. Cutri, and W. T. Reach, Near-infrared imaging and [O I] spectroscopy of IC 443 using two micron all sky survey and Infrared Space Observatory, ApJ, 547, 885-898, 2001.

Slane, P. et al., Nonthermal X-ray emission from the shell-type supernova remnant G347.3-0.5, $A p J, 525$, 357-367, 1999.

Sturner, S. J., J. G. Skibo, C. D. Dermer, and J. R. Mattox, Temporal evolution of nonthermal spectra from supernova remnants, $A p J, 490,619-632,1997$.

Sturner, S. J., C. D. Dermer, and J. R. Mattox, Are supernova remnants sources of $>100 \mathrm{MeV}$ gamma-rays?, $A \& A, 120,445-448,1996$.

Sturner, S. J., and C. D. Dermer, Association of unidentified, low-latitude EGRET sources with supernova remnants, $A \& A, 293$, L17-L20, 1995.

Tauber, J. A., R. L. Snell, R. L Dickman, and L. M. Ziurys, High-resolution images of shocked molecular clumps in the supernova remnant IC 443, $A p J, 421,570-580,1994$.

Tomkins, W., Application of likelihood analysis to $\gamma$-ray astronomy, Ph.D. thesis, Stanford University, 1999.

Wang, Z. R., I. Asaoka, S. Hayakawa, and K. Koyama, Hard X-rays from the supernova remnant IC 443, $P A S J, 44,303-308,1992$.

Yadigaroglu, I.-A., and R. W. Romani, Gamma-ray pulsars: beaming evolution, statistics, and unidentified sources, $A p J, 449,211-215,1995$.

E-mail address of S. J. Sturner sturner@swati.gsfc.nasa.gov 


\section{GODdARD SPACE FLIGHT CENTER \\ GSFC STI PUBLIC DISCLOSURE EXPORT CONTROL CHECKLIST}

The Export Control Office requests your assistance in assuring that your proposed disclosure of NASA Aeronautics and Space Administration (NASA) Scientific and Technical Information (STI) complies with the Export Administration Regulations (EAR, 15 CFR 730-774) and the International Traffic In Arms Regulations (ITAR, 22 CFR 120-130). The NASA Export Control Program requires that every domestic and international presentation/publication of Goddard Space Flight Center (GSFC) STI be reviewed through the GSFC Export Control Office in accordance with the NASA Form 1676 NASA Scientific and Technical Document Availability Authorization (DAA) process. Release of NASA information into a public forum may provide countries with interests adverse to the United States with access to NASA technology. Failure to comply with the ITAR regulations and/or the Commerce Department regulations may subject you to fines of up to $\$ 1$ million and/or up to ten years imprisonment per violation.

Completion of this checklist should minimize delays in approving most requests for presentation/publication of NASA STI.

Generally, the export of information pertaining to the design, development, production, manufacture, assembly, operation, repair, testing, maintenance or modification of defense articles, i.e., space flight hardware, ground tracking systems, launch vehicles to include sounding rockets and meteorological rockets, radiation hardened hardware and associated hardware and engineering units for these items are controlled by the State Department under the ITAR. A complete listing of items covered by the ITAR can be accessed at http://md gsfc.nasa.gov/export/home.htm. The export of information with respect to ground based sensors, detectors, high-speed computers, and national security and missile technology items are controlled by the U.S. Commerce Department under the EAR. If the information intended for release falls within the above categories but fits into one or more of the following exemptions, the information may be released.

\section{EXEMPTION I}

If your information is already in the public domain in its entirety through a non-NASA medium and/or through NASA release previously approved by the Export Control Office, the information is exempt from further review. If the information falls into this category, you may attest that you are using this exemption by signing below.

Signature

Date

\section{EXEMPTION II}

If your information pertains exclusively to the release of scientific data, i.e. data pertaining to studies of clouds, soil, vegetation, oceans, and planets, without the disclosure of information pertaining to articles controlled by the ITAR or EAR, such as flight instruments, high speed computers, or launch vehicles, the information is exempt from further review. If the information falls into this category, you may attest that you are using this exemption by signing below.
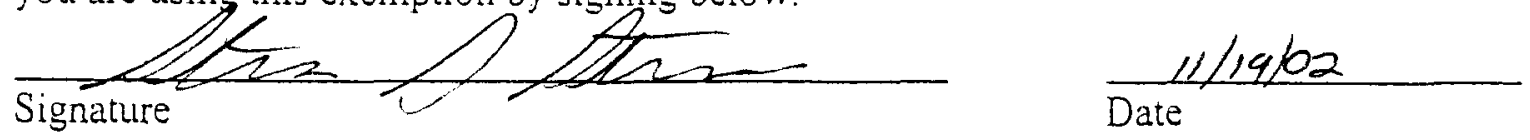

GSFC 25-49 (1/1/01) Previous editions obsolete 